\title{
Molecular gas in galaxies: much more than just the fuel of star formation
}

\section{Nicole Nesvadba ${ }^{1,2}$, Francois Boulanger ${ }^{1}$, Cinthya Herrera ${ }^{1}$, Pierre Guillard $^{3,1}$, Matt Lehnert ${ }^{4}$, Philippe Salome ${ }^{5}$, and Edith Falgarone ${ }^{6}$}

${ }^{1}$ Institut d'Astrophysique Spatiale, Universite Paris-Sud, CNRS, Centre Universitaire d'Orsay, Bat 120/121, F-91405 Orsay, France, ${ }^{2}$ email:nicole.nesvadba@ias.u-psud.fr

${ }^{3}$ Spitzer Science Center, California Institute of Technology, Pasadena, CA 91125, USA

${ }^{4}$ GEPI, Observatoire de Paris, CNRS, 92195 Meudon, France

${ }^{5}$ LERMA, Observatoire de Paris, 61 Av. de l'Observatoire, 75014, Paris, France

${ }^{6}$ LERMA, ENS Paris, CNRS, 24 rue Lhomond, 75231, Paris Cedex 05, France

\begin{abstract}
It is well known that shocks can heat molecular gas, but we are only starting to understand what the consequences might be for galaxy evolution. Observations are now revealing a growing number of galaxies undergoing phases of strong injection of kinetic energy through galaxy interactions or feedback from powerful AGN, which have bright line emission from shock-heated molecular gas that is likely powered by the dissipation of turbulent energy. These observations demonstrate that turbulence - now recognized as an important mechanism regulating star formation - may also be key in regulating galaxy growth. We report on our ongoing efforts to quantify the role of molecular gas for the dissipation of kinetic energy through observations on two very different examples, the Antennae galaxy merger and the radio galaxy 3C326 N, a site of strong mechanical AGN feedback. Both galaxies show signatures of strong dissipation of kinetic energy through shocks. We will illustrate how key parameters of these processes like bulk and turbulent velocities, gas masses, and dissipation timescales can be measured with multi-wavelength data, and how this furthers our understanding of how galaxy evolution and star formation in galaxies depend on each other.
\end{abstract}

\section{Introduction}

Galaxy interactions, mergers and feedback from star formation and active galactic nuclei are major phases in the evolution of galaxies. They all have in common that they inject large amounts of kinetic energy into the interstellar medium (ISM) of these galaxies during relatively short amounts of time compared to a Hubble time, energy which must be dissipated before stars can form. In the classical galaxy evolution scenarios, most of this energy is thermalized and produces extended halos of hot $\left(10^{7} \mathrm{~K}\right) \mathrm{X}$-ray emitting plasma. Recent Spitzer observations of bright, shock-heated $\mathrm{H}_{2}$ line emission in an increasing number of galaxies (typically interacting galaxies or the hosts of powerful radio-loud active galactic nuclei, Guillard et al., 2012, Ogle et al., 2010) however paint a more nuanced picture, where large fractions of the kinetic energy are not thermalized, but feed a turbulent cascade which pervades the ISM. Guillard et al. (2009) propose a scenario where turbulence drives a mass and energy cycle across the phases of the ISM, which involves the formation of $\mathrm{H}_{2}$ from the warm neutral medium. This implies repeated heating of molecular gas, which is associated with the dissipation of kinetic energy. This boosts line luminosities and makes molecular gas an important, so far largely ignored, coolant of the ISM. The turbulent energy is ultimately dissipated through bright line emission, in particular pure-rotational lines of warm $\mathrm{H}_{2}$ seen in the mid-infrared with Spitzer-IRS spectroscopy. 
To further elucidate the role of turbulence in galaxies with emission lines of warm $\mathrm{H}_{2}$, we have conducted a number of detailed observations of particularly suited individual galaxies in recent years, including the intergalactic shock in Stephan's Quintet (Guillard et al., 2009, 2010, 2012b), as well as the nearby radio galaxy 3C326 N (Nesvadba et al. 2010, 2011) and the Antennae galaxy merger (Herrera et al., 2011, 2012). Observationally we rely on spectroscopy of pure-rotational $\mathrm{H}_{2}$ lines measured with Spitzer, ro-vibrational $\mathrm{H}_{2}$ and ionized gas lines seen at higher spatial and spectral resolution, e.g., with the VLT/SINFONI imaging spectrograph, and CO rotational lines measured with millimeter interferometers including ALMA (Herrera et al. 2012).

Emission-line diagnostics in several wavebands show that the diffuse, extended gas in all these systems is heated by the dissipation of turbulence, not UV photons from star formation or X-rays from the active galactic nucleus. Comparison with models of interstellar magnetized shocks by Flower \& Pineau des Forets (2010) and Lesaffre et al. (2012) demonstrates that the $\mathrm{H}_{2}$ line ratios are consistent with C-type shocks with a range of velocities of few to few $10 \mathrm{sm} \mathrm{s}^{-1}$. Shock velocities inferred from the measured $\mathrm{H}_{2}$ line ratios are lower than the bulk velocities on the scales where the kinetic energy is injected, consistent with an energy cascade from large to smaller scales (Guillard et al. 2009, Lesaffre et al. 2012).

For the cases of the radio galaxy 3C326 N and the Antennae galaxy merger we will now illustrate how a detailed observational analysis of the turbulent dissipation of kinetic energy in the molecular gas of galaxies can significantly further our understanding of how star formation and galaxy growth are regulated during key phases of galaxy evolution.

\section{Dense gas without star formation: the kpc-sized turbulent molecular disk in 3C $326 \mathrm{~N}$}

3C326 $\mathrm{N}$ is a "textbook" elliptical galaxy with an "old, red, and dead" stellar population of mass $\mathrm{M}_{\text {stellar }}=3 \times 10^{11} \mathrm{M}_{\odot}$ formed more than $10 \mathrm{Gyr}$ ago. Stellar absorption lines in the optical show that less than $10^{6} \mathrm{M}_{\odot}$ of stars may have formed in the last $\geqslant 300$ Myr (Nesvadba et al. 2011), and the current star-formation rate estimated from Spitzer $70 \mu \mathrm{m}$ photometry is very low, $\mathrm{SFR}<0.07 \mathrm{M}_{\odot} \mathrm{yr}^{-1}$ (Ogle et al., 2007). 3C326 $\mathrm{N}$ has one of the largest, oldest radio jets on the sky, with an age of $60-200 \mathrm{Myr}$, and a mechanical power of $10^{44-45} \mathrm{erg} \mathrm{s}^{-1}$ (see Nesvadba et al. 2010, 2011 and references therein). Its pure-rotational $\mathrm{H}_{2}$ luminosity is outstanding, $10^{42} \mathrm{erg} \mathrm{s}^{-1}$, a factor 100 greater than the X-ray luminosity of the AGN (Ogle et al., 2007), and likewise, star formation is not powerful enough to heat the gas. This leaves the radio source as the sole plausible source of kinetic energy in this galaxy.

Comparison of Spitzer data and IRAM CO(1-0) millimeter interferometry shows that at least about half of the $2 \times 10^{9} \mathrm{M}_{\odot}$ of molecular gas in 3C326 N (for a Milky-Way-like $\mathrm{X}$-factor) is heated to temperatures $>100 \mathrm{~K}$, which requires the on-going injection of kinetic energy. 3C326 $\mathrm{N}$ has a wind seen in Na D absorption and through broad, blueshifted wings of ionized and warm molecular Hydrogen lines, with a terminal velocity of -1800 $\mathrm{km} \mathrm{s}^{-1}$ and mass and energy outflow rates of few $10 \mathrm{~s} \mathrm{M}_{\odot} \mathrm{yr}^{-1}$ and about $10^{43} \mathrm{erg} \mathrm{s}^{-1}$, respectively. The only plausible driver of this outflow is the radio jet, star formation and AGN bolometric power are too weak (see Nesvadba et al. 2010 for details). The molecular gas is in a $3 \mathrm{kpc}$ disk of high mass surface density, $\Sigma=250 \mathrm{M}_{\odot} \mathrm{pc}^{2}$, and remarkably broad (rotation-corrected) $\mathrm{H}_{2}$ lines with FWHM $=400-600 \mathrm{~km} \mathrm{~s}^{-1}$ across the disk. Line profiles show multiple velocity components as expected from stirred-up, perhaps turbulent gas. Broad line widths have also been found in integrated mid-infrared $\mathrm{H}_{2}$ spectra of other nearby radio galaxies (Guillard et al., 2012). 


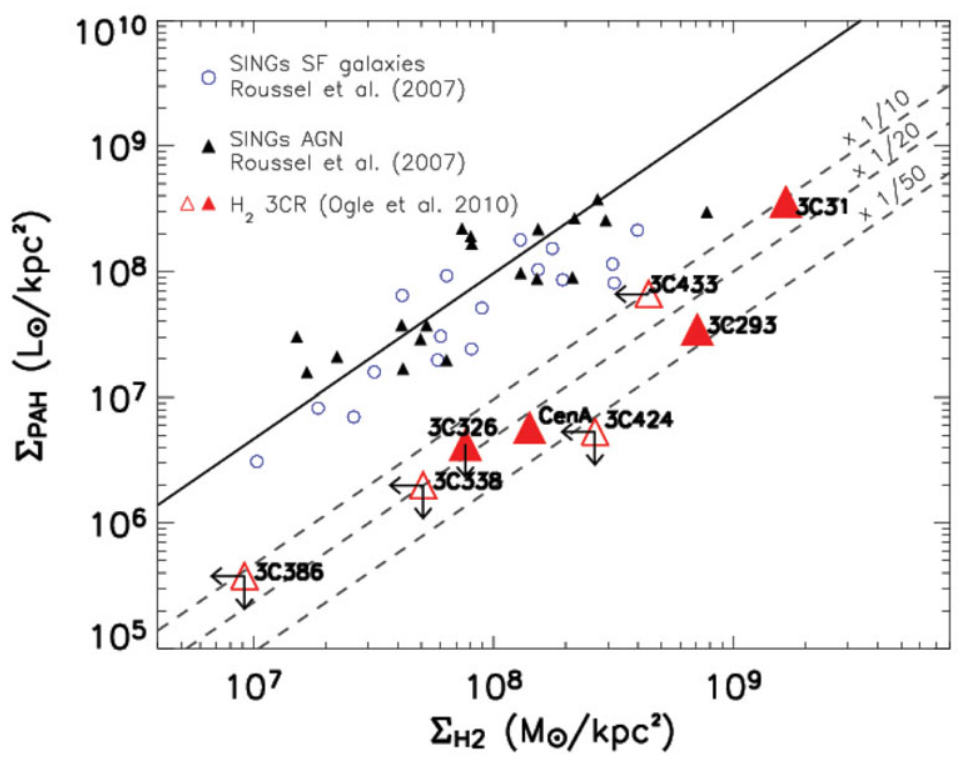

Figure 1. Surface brightness of the $7.7 \mu \mathrm{m}$ PAH feature as a function of molecular gas mass surface density measured from $\mathrm{CO}(1-0)$ for "ordinary" star-forming galaxies from the SINGS survey (small symbols) and powerful radio galaxies with extended molecular disks seen in CO (large symbols). Under the assumption that PAH surface brightness probes star-formation intensity, the offset between radio and SINGSs galaxies implies that star-formation efficiencies in radio galaxies with powerful AGN and bright $\mathrm{H}_{2}$ line emission of warm molecular gas are lowered by factors 10-50. For individual galaxies like 3C326 N or 3C293, which have far-infrared photometry, star-formation estimates from warm dust suggest similar offsets. See Nesvadba et al. (2010) for details.

The outflow in 3C326 $\mathrm{N}$ is the first found in an AGN host with no evidence of ongoing star formation, but more remarkable are the low star-formation rates. The gas mass surface density of $3 \mathrm{C} 326 \mathrm{~N}$ is akin to that measured on similar scales in LIRGs, but the star formation intensity is a factor $\sim 60$ lower, leading to a pronounced offset between $3 \mathrm{C} 326 \mathrm{~N}$ (and other radio galaxies with bright $\mathrm{H}_{2}$ line emission) and 'ordinary' star-forming galaxies in the Schmidt-Kennicutt diagram (Fig. 1).

Nesvadba et al. (2011) propose that the high turbulence produced by the jet in the ISM of $3 \mathrm{C} 326 \mathrm{~N}$ could be the reason why this galaxy is not forming stars, in spite of the high-surface density molecular disk. The low star-formation rates in 3C326 N would thus be the consequence of mechanical feedback from the active galactic nucleus, in line with current theories of star formation, which attribute a major role to turbulence in setting the star-formation efficiency of molecular clouds (Krumholz \& McKee 2005). Star-forming molecular clouds are in a near-equilibrium between turbulent $\left(E_{\text {turb }}\right)$ and gravitational potential energy $\left(E_{\text {grav }}\right)$, which is commonly expressed as the "virial" parameter $\alpha_{v i r}=$ $2 E_{\text {turb }} / E_{\text {grav }} \sim 1$. Only molecular clouds that are near this critical value will form stars. The distance of $3 \mathrm{C} 326 \mathrm{~N}$ at $\mathrm{z}=0.1$ currently prevents us from measuring the line dispersions directly on the scales of $<100 \mathrm{pc}$ relevant for molecular cloud collapse and star formation, however, we can extrapolate from the spatially resolved scales of $\sim 1 \mathrm{kpc}$ downwards (Fig. 4 of Nesvadba et al. 2011). We find that $\alpha_{v i r}$ in $3 \mathrm{C} 326 \mathrm{~N}$ is about an order of magnitude greater than that of marginally bound molecular clouds on all scales from the $\sim 1 \mathrm{kpc}$ that are resolved and down to few pc, if the size-velocity relationship of turbulent gas applies in 3C326 N (e.g., Larson et al. 1981), i.e., if the gas motion indeed 


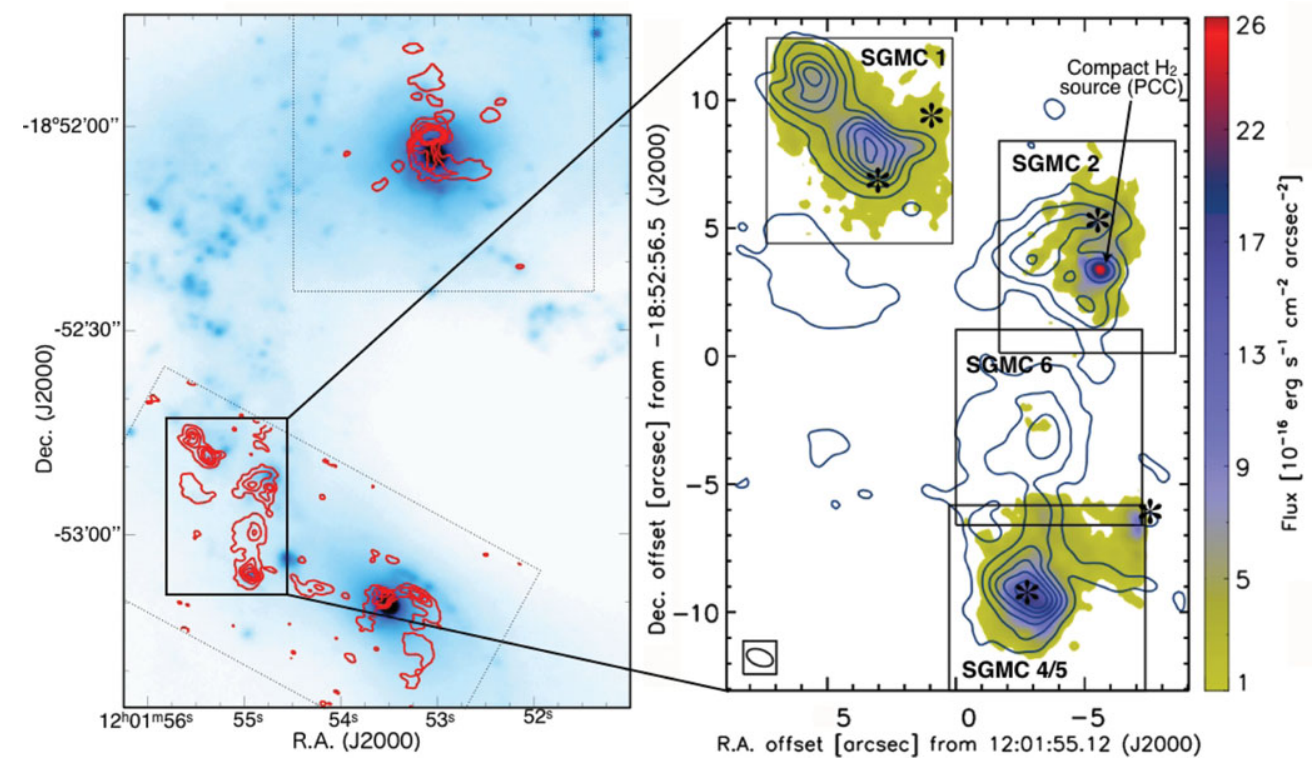

Figure 2. (Left) CO morphology of the Antennae measured with ALMA (contours) shown on top of our K-band continuum image (Herrera et al. 2011, 2012). The dotted boxes show the area covered by ALMA, the solid box highlights the overlap region. (Right) $\mathrm{H}_{2}$ 1-0 S(1) morphology observed with SINFONI. Boxes show the SINFONI field of view for each of our four pointings. Contours show the $\mathrm{CO}(3-2)$ morphology. The positions of super-star clusters are marked with asterisks, and we also label the compact $\mathrm{H}_{2}$ source PCC 1 (see text and Herrera et al., 2012 for details.)

follows a turbulent cascade. This finds support from the observed mass-to-light ratio of $\mathrm{H}_{2}$ (see Nesvadba et al. 2011 for details), and would suggest that the gas is overall so far from being bound that it is impossible to assemble enough mass even in the densest regions of the disk to form gravitationally bound clouds and enable star formation. Obviously, this scenario can be tested further with high-resolution observations, once ALMA has reached its full potential.

\section{From large scale gas compression to cluster formation in the Antennae overlap region}

Our second example is the nearby archetypal galaxy merger "The Antennae". Major mergers are the sites of the most intense star formation in the local Universe, with the potential of turning gas-rich spiral galaxies into "red, and dead" elliptical galaxies within a few 100 Myr. Most stars in galaxy mergers form in super-star clusters with masses of up to $10^{7} \mathrm{M}_{\odot}$. Hydrodynamic merger models suggest that large complexes of cold gas (super-giant molecular complexes, SGMCs) form where convergent gas flows trigger the compression, cooling, and gravitational fragmentation of the gas (Teyssier et al. 2010). Within SGMCs, a hierarchy of structures must form, including clouds massive enough to form super-star clusters (Weidner et al. 2010). Developing a physical understanding of how merger-driven convergent flows may regulate star formation in galaxy mergers has so far been the domain of numerical models, with few direct, observational tests.

The Antennae galaxy pair (NGC4038/39) at a distance of only $22 \mathrm{Mpc}$ is one of the few major mergers on the sky where we reach the spatial resolution $<<100 \mathrm{pc}$ and sensitivity necessary for such tests. Most molecular gas in the Antennae is in the "overlap region" 
where both galaxies interact with each other, forming a complex of super-giant molecular clouds recently observed with ALMA at sub-arcsecond resolution (Herrera et al. 2012). We use the maps of $\mathrm{CO}(3-2)$ seen with ALMA with those of the $\mathrm{v}=1-0 \mathrm{H}_{2}$ lines seen with VLT/SINFONI to compare a tracer of the bulk mass with a tracer of the kinetic energy dissipation. Both show very similar kinematics and spatial distributions, although their flux ratio varies by up to about an order of magnitude (Fig. 2; Herrera et al. 2012). The morphologies of the warm $\mathrm{H}_{2}$ and line ratios show that the gas is overall not associated with super-star clusters (SSCs) or heated by UV photons from young stars, except in the SSCs themselves.

The brightest source of warm $\mathrm{H}_{2}$ line emission in the Antennae, PCC 1, is however very compact, with about $50 \mathrm{pc}$ diameter and a $\mathrm{CO}$ line width of $90 \mathrm{~km} \mathrm{~s}^{-1}$. PCC 1 is not associated with any SSC, and has a very peculiar NIR spectrum showing no $\mathrm{Br} \gamma$ emission from ionized Hydrogen, with a $\mathrm{H}_{2} 1-0 \mathrm{~S}(1) / \mathrm{Br} \gamma$ ratio $>100$ (Herrera et al. 2011). The low $\mathrm{Br} \gamma$ flux and faint $\mathrm{GHz}$ radio continuum at the position of PCC 1 suggest that stars contribute $\leqslant 0.1 \%$ of the $\sim 10^{7} \mathrm{M}_{\odot}$ virial mass of this object. PCC 1 is at the position of the largest $\mathrm{CO}(3-2)$ velocity gradient in the entire overlap region, consistent with expectations for a cloud whose growth is fed by convergent gas flows driven by the interaction of NGC4038 and NGC4039. Its size and virial mass estimate are consistent with what would be expected from a cloud which is about to form a massive (few $10^{6}$ $\left.\mathrm{M}_{\odot}\right)$ super-star cluster.

The enormous luminosity of the lines of warm $\mathrm{H}_{2}$, which is also seen at lower spatial resolution in the pure-rotational $\mathrm{H}_{2}$ lines with Spitzer/IRS, and shock-like line ratios in the NIR suggest that PCC 1 is dissipating kinetic energy very rapidly on a timescale of a few $10^{6}$ yrs. The source of kinetic energy may either be accretion of gas onto PCC 1 , or the contraction of PCC 1 itself (Herrera et al. 2012). In either case, this can interpreted as a signature of how turbulent gas driven by the galaxy interaction is settling into a gravitationally bound cloud which may be about to start forming stars. We are not aware that a similar case would have previously been discussed in the literature.

Taken together, the diffuse and compact $\mathrm{H}_{2}$ line emission in the Antennae overlap region paint a scenario that is consistent overall with star-cluster formation fed by convergent gas flows driven by the galaxy interaction. The interaction would remain compressive on $\mathrm{kpc}$ scales for few $10^{7} \mathrm{yrs}$, long enough for dense clouds akin to PCC 1 to lose their turbulent energy, settle, form gravitationally bound clouds, and then super-star clusters. In contrast, the kinetic energy injection into the diffuse gas appears to be large enough to balance turbulent dissipation over much longer timescales, of order $10^{8}$ yrs. This is longer than the crossing time of the two spiral arms of NGC4038/39. Most of the gas in the super-giant molecular cloud complexes may therefore plausibly disperse at the end of the passage of the two disks without forming stars. This is consistent with the low star-formation efficiencies that we find in the overlap region, of order $1 \%$ and akin to molecular clouds in the Milky Way (Herrera et al. 2011).

Overall this suggests that turbulence may play an important role in balancing gravity and regulating star formation during galaxy interactions, a role that has so far been mainly attributed to feedback from star formation. In turn, our observations deemphasize the role of feedback, in particular during the phases of gas collapse that precede the formation of young stars, where feedback is thus implausible. Our on-going work suggests that turbulence fed by the galaxy interaction may enhance the effects of feedback once the super-star cluster has formed, as much of the gas is likely to be only marginally gravitationally bound, making it easier to remove through the additional energy injection from feedback processes (Herrera et al. 2013, in prep.). 


\section{Conclusions}

Our observations of objects as diverse as powerful radio galaxies and gas-rich major mergers illustrate that turbulent heating of the interstellar medium in galaxies may play an important role in regulating star formation during critical phases of galaxy evolution that are characterized by the strong injection of kinetic energy into the interstellar medium. In either example, turbulence plays a major role in regulating star formation, in contrast to many models of galaxy evolution, which attribute this role solely to gas removal through winds. Our observations rather suggest that wind and turbulence are two intricately related processes which together regulate galaxy growth.

\section{References}

Flower, D. R. \& Pineau des Forets, G. 2010, MNRAS, 406, 1745

Guillard, P., et al. 2009, A\&SA, 502, 515

Guillard, P., et al. 2010, A\&A, 518, 59

Guillard, P., et al. 2012a, ApJ, 747, 95

Guillard, P., et al. 2012b, ApJ, 749, 158

Herrera, C. N., Boulanger, F., \& Nesvadba, N. P. H. 2011, A\& A, 534, 138

Herrera, C. N., et al. 2012, A\& $A, 538$,L9

Krumholz, M. R. \& McKee, C. F. 2005, ApJ, 630, 250

Lesaffre, P., et al. 2012, A\&A, accepted

Nesvadba, N. P. H., et al. 2010, A\&A, 521, 65

Nesvadba, N. P. H., et al. 2011, A\& $A, 536$, L5

Ogle, P., et al. 2007, ApJ, 668, 699

Ogle, P., et al. 2010, ApJ, 724, 1193

Teyssier, R., Chapon, D., \& Bournaud, F. 2010, ApJ, 720, L149

Weidner, C., Bonnell, I. A., \& Zinnecker, H. 2010, ApJ, 724, 1503 